\title{
GEOMETRICAL INEQUALITIES IN ACUTE TRIANGLES INVOLVING THE MEDIANS IX
}

\author{
Béla FINTA \\ „Petru Maior" University of Tîrgu Mureş \\ Nicolae Iorga Street, no. 1, 540088, Tîrgu Mureş, Romania \\ e-mail: fintab@science.upm.ro
}

\begin{abstract}
The purpose of this paper is to give a positive answer to a new open question that aimed to generalize previous open questions formulated by researchers in the field of geometrical inequalities. In this sense we prove that in every acute triangle ABC from $a<b<c$ result $a^{\alpha}+m_{a}^{\alpha}<b^{\alpha}+m_{b}^{\alpha}<c^{\alpha}+m_{c}^{\alpha}$, where $\alpha \in\left(2,2 \cdot \log _{\frac{5}{4}} \frac{5}{3}\right)$.
\end{abstract}

Keywords: geometrical inequalities, acute triangle, medians, bisectrices, altitudes

\section{Introduction}

Let us consider the acute triangle $\mathrm{ABC}$ with sides $a=B C, b=A C$ and $c=A B$. In [1] appeared the following open question due to Pál Erdős: "if ABC is an acute triangle such that $a<b<c$ then $a+l_{a}<$ $b+l_{b}<c+l_{c}$ ", where $l_{a}, l_{b}, l_{c}$ means the length of the interior bisectrices corresponding to the sides $\mathrm{BC}$, $\mathrm{AC}$ and $\mathrm{AB}$, respectively.

In [2] Mihály Bencze proposed the following open question, which is a generalization of the problem of Pál Erdős: "determine all points $M \in \operatorname{Int}(A B C)$, for which in case of $B C<C A<A B$ we have $C B+A A^{\prime}<C A+B B^{\prime}<A B+C C^{\prime}$, where $\mathrm{A}^{\prime}, \mathrm{B}^{\prime}, \mathrm{C}^{\prime}$ is the intersection of $\mathrm{AM}, \mathrm{BM}, \mathrm{CM}$ with sides $\mathrm{BC}, \mathrm{CA}, \mathrm{AB}$ ". Here with $\operatorname{Int}(A B C)$ we denote the interior points of the triangle $\mathrm{ABC}$.

If we try for "usual" acute triangles $\mathrm{ABC}$, we can verify the validity of the Erdôs inequality. But in [3] we realized to obtain an acute triangle for which the Erdôs inequality is false: let $\mathrm{ABC}$ be such that $c=10+\epsilon, b=10$ and $a=1$, where $\epsilon>0$ is a "very small" positive quantity. Using the trigonometrical way combined with some elementary properties from algebra and mathematical analysis we showed that for this "extreme" acute triangle from $a<b<c$ results $c+l_{c}<b+l_{b}$.
In [4] József Sándor proved some new geometrical inequalities using in the open question of Pál Erdős instead of bisectrices altitudes and medians.

In [5] Károly Dáné and Csaba Ignát studied the open question of Mihály Bencze using the computer.

In connection with Erdős's problem we formulated the following open question: "if $\mathrm{ABC}$ is an acute triangle such that $a<b<c$ then $a^{2}+l_{a}^{2}<b^{2}+l_{b}^{2}<$ $c^{2}+l_{c}^{2}$ ". In [6] we proved the validity of this statement.

At the same time we formulated another new open question: "if $\mathrm{ABC}$ is an acute triangle such that $a<$ $b<c$ then $a^{4}+l_{a}^{4}<b^{4}+l_{b}^{4}<c^{4}+l_{c}^{4}$ ". In [7] we realized to find two acute triangles ABC such that in the first triangle from $a<b<c(b=a+\epsilon, c=a+$ $2 \epsilon$ with $\epsilon>0$ a small positive quantity) we obtained $a^{4}+l_{a}^{4}<b^{4}+l_{b}^{4}$, but in the second triangle from $a<b<c(b=a+\epsilon, c=a \sqrt{2}$ with $\epsilon>0$ a small positive quantity) we deduced $a^{4}+l_{a}^{4}>b^{4}+l_{b}^{4}$. So the answer to our question is negative.

Next we denote by $h_{a}, h_{b}$ and $h_{c}$ the length of the altitudes of the triangle $\mathrm{ABC}$, which correspond to the sides $\mathrm{BC}, \mathrm{CA}$ and $\mathrm{AB}$, respectively. Then we can formulate the following more general similar question, replacing in the open question of Pál Erdős the bisectrices with altitudes: "if $\mathrm{ABC}$ is an acute triangle such

(c) 2015 Published by "Petru Maior" University Press. This is an open access article under the CC BY-NC-ND license (http://creativecommons.org/licenses/by-nc-nd/4.0/). 
that $a<b<c$ then $a^{\alpha}+h_{a}^{\alpha}<b^{\alpha}+h_{b}^{\alpha}<c^{\alpha}+h_{c}^{\alpha}$, where $\alpha \in \mathbb{R}$ is a real number". In [4] or [8] there is showed, that this property is true for all $\alpha \in \mathbb{R}^{*}$, where $\mathbb{R}^{*}=\mathbb{R}-\{0\}$.

Next we denote by $m_{a}, m_{b}$ and $m_{c}$ the length of the medians, which correspond to the sides $\mathrm{BC}, \mathrm{CA}$ and $\mathrm{AB}$, respectively. Then we can formulate the following more general similar question, replacing in the open question of Pál Erdős the bisectrices with medians: "if $A B C$ is an acute triangle from $a<b<c$ it results $a^{\alpha}+m_{a}^{\alpha}<b^{\alpha}+m_{b}^{\alpha}<c^{\alpha}+m_{c}^{\alpha}$, where $\alpha \in \mathbb{R}^{\prime}$.

We mention, that this property is not obvious, because $a<b \Leftrightarrow a^{2}<b^{2} \Leftrightarrow 2\left(a^{2}+c^{2}\right)-b^{2}<$ $2\left(b^{2}+c^{2}\right)-a^{2} \Leftrightarrow \sqrt{\frac{2\left(a^{2}+c^{2}\right)-b^{2}}{4}}<\sqrt{\frac{2\left(b^{2}+c^{2}\right)-a^{2}}{4}} \Leftrightarrow$ $m_{b}<m_{a}$.

Similarly $b<c \Leftrightarrow m_{b}>m_{c}$.

If $\alpha=0$, then is immediately that our problem is false.

The above proposed problem is solved in [8] for $\alpha \in\{1,2,4\}$ and we obtained that for $\alpha=1$ it is false and for $\alpha=2$ and $\alpha=4$ it is true. In [9] we showed for $\alpha=8$, that our question is false, and in [10] we proved that for $\alpha=-2$ our inequality is false, too. In [11] we obtained for $\alpha=2 n, n \in \mathbb{N}$, $n \geq 3$, even natural numbers that our statement is false. In [12] we showed for $\alpha=2 n+1, n \in \mathbb{N}$, $n \geq 2$ odd natural numbers that our affirmation is false. In [13] we proved for $\alpha=-2 n, n \in \mathbb{N}^{*}$, negative even integer numbers, that our statement is not valid. In [14] we proved for $\alpha=-2 n-1, n \in \mathbb{N}$, negative even integer numbers, that our statement is not valid. In [15] we showed that our open question is false for every positive rational number $\alpha=\frac{p}{q} \in \mathbb{Q}_{+}$ and $\alpha=\frac{p}{q}>\log _{\frac{5}{4}}\left(\frac{5}{3}\right)^{2}$. In [16] we showed that our open question is false for every negative rational number $\alpha=\frac{p}{q} \in \mathbb{Q}_{-}$.

\section{Main part}

The purpose of this paper is to study this open question for $\alpha \in\left(2,2 \cdot \log _{\frac{5}{4}} \frac{5}{3}\right)$ and we obtain

Proposition 1. If $\alpha \in\left(2,2 \cdot \log _{\frac{5}{4}} \frac{5}{3}\right)$ then for every acute triangle $A B C$ from $a<b^{4}<c$ results $a^{\alpha}+$ $m_{a}^{\alpha}<b^{\alpha}+m_{b}^{\alpha}<c^{\alpha}+m_{c}^{\alpha}$.

Proof. Using the cosine theorem $c^{2}=a^{2}+b^{2}-2$. $a \cdot b \cdot \cos C$ the triangle $A B C$ is acute if and only if $c^{2}<a^{2}+b^{2}$. This means $a<b<c<\sqrt{a^{2}+b^{2}}$. We divide these inequalities by $a$ and we denote $x=$ $\frac{b}{a}, y=\frac{c}{a}$, so we get $1<x<y<\sqrt{1+x^{2}}$. We have the following sequence of equivalent inequalities: $a^{\alpha}+m_{a}^{\alpha}<b^{\alpha}+m_{b}^{\alpha} \Leftrightarrow a^{\alpha}+\left(\frac{\sqrt{2\left(b^{2}+c^{2}\right)-a^{2}}}{2}\right)^{\alpha}<$ $b^{\alpha}+\left(\frac{\sqrt{2\left(a^{2}+c^{2}\right)-b^{2}}}{2}\right)^{\alpha} \Leftrightarrow 1+\frac{\left[2\left(x^{2}+y^{2}\right)-1\right]^{\frac{\alpha}{2}}}{2^{\alpha}}<x^{\alpha}+$ $\frac{\left[2\left(1+y^{2}\right)-x^{2}\right]^{\frac{\alpha}{2}}}{2^{\alpha}}$. For a fixed $y>1$ we consider the function $f:[1, y] \rightarrow \mathbb{R}, f(x)=x^{\alpha}-1+\frac{\left[2\left(1+y^{2}\right)-x^{2}\right]^{\frac{\alpha}{2}}}{2^{\alpha}}-$ $\frac{\left[2\left(x^{2}+y^{2}\right)-1\right]^{\frac{\alpha}{2}}}{2^{\alpha}}$. We will show $f^{\prime}(x)>0$ for every $x \in(1, y)$, which means that $\mathrm{f}$ is strictly monotone increasing function, so $f(x)>f(1)=0$ and our inequality is true. We get $f^{\prime}(x)>0 \Leftrightarrow \alpha \cdot x^{\alpha-1}+\frac{\alpha}{2}$. $\frac{\left[2\left(1+y^{2}\right)-x^{2}\right]^{\frac{\alpha}{2}-1}}{2^{\alpha}} \cdot(-2 x)-\frac{\alpha}{2} \cdot \frac{\left[2\left(x^{2}+y^{2}\right)-1\right]^{\frac{\alpha}{2}-1}}{2^{\alpha}} \cdot(4 x)>$ $0 \Leftrightarrow 2^{2^{\alpha}} \cdot x^{\alpha-2}>\left[2\left(1+y^{2}\right)-x^{2}\right]^{\frac{\alpha}{2}-1}+\left[2\left(x^{2}+\right.\right.$ $\left.\left.y^{2}\right)-1\right]^{\frac{\alpha}{2}-1} \cdot 2$. But we have $2\left(1+y^{2}\right)-x^{2}<$ $2\left(1+1+x^{2}\right)-x^{2}=4+x^{2}<4 x^{2}+x^{2}=5 x^{2}$ and $2\left(x^{2}+y^{2}\right)-1<2\left(x^{2}+1+x^{2}\right)-1=4 x^{2}+1<$ $4 x^{2}+x^{2}=5 x^{2}$. In order to prove the inequality $2^{\alpha} \cdot x^{\alpha-2}>\left[2\left(1+y^{2}\right)-x^{2}\right]^{\frac{\alpha}{2}-1}+\left[2\left(x^{2}+y^{2}\right)-\right.$ $1]^{\frac{\alpha}{2}-1} \cdot 2$ is enough to show the validity of the inequality $2^{\alpha} \cdot x^{\alpha-2}>\left(5 \cdot x^{2}\right)^{\frac{\alpha}{2}-1}+\left(5 \cdot x^{2}\right)^{\frac{\alpha}{2}-1} \cdot 2 \Leftrightarrow 2^{\alpha}>$ $(\sqrt{5})^{\alpha-2}+(\sqrt{5})^{\alpha-2} \cdot 2 \Leftrightarrow \alpha<2 \cdot \log _{\frac{5}{4}} \frac{5}{3}$. Consequently if $\alpha<2 \cdot \log _{\frac{5}{4}} \frac{5}{3}$ then results $a^{\alpha}+m_{a}^{\alpha}<$ $b^{\alpha}+m_{b}^{\alpha}$.

We have the following sequence of equivalent inequalities: $b^{\alpha}+m_{b}^{\alpha}<c^{\alpha}+m_{c}^{\alpha} \Leftrightarrow b^{\alpha}+$ $\left(\frac{\sqrt{2\left(a^{2}+c^{2}\right)-b^{2}}}{2}\right)^{\alpha}<c^{\alpha}+\left(\frac{\sqrt{2\left(a^{2}+b^{2}\right)-c^{2}}}{2}\right)^{\alpha} \Leftrightarrow x^{\alpha}+$ $\frac{\left[2\left(1+y^{2}\right)-x^{2}\right]^{\frac{\alpha}{2}}}{2^{\alpha}}<y^{\alpha}+\frac{\left[2\left(1+x^{2}\right)-y^{2}\right]^{\frac{\alpha}{2}}}{2^{\alpha}}$. For a fixed $y>1$ we consider the function $f:[1, y] \rightarrow \mathbb{R}, f(x)=$ $y^{\alpha}-x^{\alpha}+\frac{\left[2\left(1+x^{2}\right)-y^{2}\right]^{\frac{\alpha}{2}}}{2^{\alpha}}-\frac{\left[2\left(1+y^{2}\right)-x^{2}\right]^{\frac{\alpha}{2}}}{2^{\alpha}}$. We will show $f^{\prime}(x)<0$ for every $x \in(1, y)$, which means that $\mathrm{f}$ is strictly monotone decreasing function, so $f(x)>f(y)=0$ and our inequality is true. We get $f^{\prime}(x)<0 \Leftrightarrow-\alpha \cdot x^{\alpha-1}+\frac{\alpha}{2} \cdot \frac{\left[2\left(1+x^{2}\right)-y^{2}\right]^{\frac{\alpha}{2}-1}}{2^{\alpha}} \cdot(4 x)-$ $\frac{\alpha}{2} \cdot \frac{\left[2\left(1+y^{2}\right)-x^{2}\right]^{\frac{\alpha}{2}-1}}{2^{\alpha}} \cdot(-2 x)<0 \Leftrightarrow 2^{\alpha} \cdot x^{\alpha-2}>$ $\left[2\left(1+x^{2}\right)^{2^{\alpha}}-y^{2}\right]^{\frac{\alpha}{2}-1} \cdot 2+\left[2\left(1+y^{2}\right)-x^{2}\right]^{\frac{\alpha}{2}-1}$. But we have $2\left(1+x^{2}\right)-y^{2}<2\left(1+x^{2}\right)-x^{2}=$ $2+x^{2}<2 x^{2}+x^{2}=3 x^{2}$ and $2\left(1+y^{2}\right)-x^{2}<$ $2\left(1+1+x^{2}\right)-x^{2}=4+x^{2}<4 x^{2}+x^{2}=5 x^{2}$. In order to prove the inequality $2^{\alpha} \cdot x^{\alpha-2}>\left[2\left(1+x^{2}\right)-\right.$ $\left.y^{2}\right]^{\frac{\alpha}{2}-1} \cdot 2+\left[2\left(1+y^{2}\right)-x^{2}\right]^{\frac{\alpha}{2}-1}$ is enough to show the validity of the inequality $2^{\alpha} \cdot x^{\alpha-2}>\left(3 x^{2}\right)^{\frac{\alpha}{2}-1}$. $2+\left(5 x^{2}\right)^{\frac{\alpha}{2}-1} \Leftrightarrow 2^{\alpha}>2 \cdot(\sqrt{3})^{\alpha-2}+(\sqrt{5})^{\alpha-2}$. For $\alpha>2$ we get $(\sqrt{3})^{\alpha-2}<(\sqrt{5})^{\alpha-2}$, so the inequality $2^{\alpha}>2 \cdot(\sqrt{5})^{\alpha-2}+(\sqrt{5})^{\alpha-2}$ implies the inequality $2^{\alpha}>2 \cdot(\sqrt{3})^{\alpha-2}+(\sqrt{5})^{\alpha-2}$. But the solution of the inequality $2^{\alpha}>2 \cdot(\sqrt{5})^{\alpha-2}+(\sqrt{5})^{\alpha-2}$ is $\alpha<2 \cdot \log _{\frac{5}{4}} \frac{5}{3}$. Consequently if $\alpha \in\left(2,2 \cdot \log _{\frac{5}{4}} \frac{5}{3}\right)$ then results $b^{\alpha}+m_{b}^{\alpha}<c^{\alpha}+m_{c}^{\alpha}$.

\section{Discussion and conclusion}

Conclusion Our open question is false for $\alpha=0$, $\alpha=1$, for every positive rational number $\alpha=\frac{p}{q} \in$ $\mathbb{Q}_{+} \cap\left(\log _{\frac{5}{4}}\left(\frac{5}{3}\right)^{2},+\infty\right)$ and for every negative rational number $\alpha=\frac{p}{q} \in \mathbb{Q}_{-}$, but is true for $\alpha \in$ $\left[2,2 \cdot \log _{\frac{5}{4}} \frac{5}{3}\right)$. In the next paper we will study our inequality for $\alpha$ irrational number. 


\section{References}

[1] Open Question OQ.14, Mathematical Magazine Octogon, Vol. 3, No. 1, 1995, pp. 54, Braşov, Romania

[2] Open Question OQ.27, Mathematical Magazine Octogon, Vol. 3, No. 2, 1995, pp. 64, Braşov, Romania

[3] Béla Finta, Solution for an Elementary Open Question of Pál Erdős, Mathematical Magazine Octogon, Vol. 4, No. 1, 1996, pp. 74-79, Braşov, Romania

[4] József Sándor, On Some New Geometric Inequalities, Mathematical Magazine Octogon, Vol. 5, No. 2, 1997, pp. 66-69, Braşov, Romania

[5] Károly Dáné, Csaba Ignát, On the Open Question of P. Erdős and M. Bencze, Mathematical Magazine Octogon, Vol. 6, No. 1, 1998, pp. 73-77, Braşov, Romania

[6] Béla Finta, A New Solved Question in Connection to a Problem of Pál Erdôs, Proceedings of the 3rd Conference on the History of Mathematics and Teaching of Mathematics, University of Miskolc, May 21-23, 2004, pp. 56-60, Miskolc, Hungary

[7] Béla Finta, A New Solved Question in Connection to a Problem of Pál Erdős II, Didactica Matematicii, "Babeş-Bolyai" University, Vol. 24, 2006, pp. 65-70, Cluj-Napoca, Romania

[8] Béla Finta, Some Geometrical Inequalities in Acute Triangle, Lucrările celei de a III-a Conferinţe anuale a Societăţii de Ştiinţe Matematice din România, Vol. 3, Comunicări metodicoştiinţifice, Universitatea din Craiova, 26-29 mai 1999, pp.193-200, Craiova, România

[9] Béla Finta, Geometrical Inequalities in Acute Triangle Involving the Medians, Didactica Matematicii, "Babeş-Bolyai" University, Vol. 22, 2004, pp. 131-134, Cluj-Napoca, Romania
[10] Béla Finta, Geometrical Inequalities in Acute Triangle Involving the Medians II, Didactica Matematicii, "Babeş-Bolyai" University, Vol. 25, No. 1, 2007, pp. 75-77, Cluj-Napoca, Romania

[11] Béla Finta, Zsuzsánna Finta, Geometrical Inequalities in Acute Triangle Involving the Medians III, International Conference "Mathematical Education in the Current European Context", 3rd edition, November 23, 2012, Braşov, Romania, pp. 151-156, ISBN 978-606-624-475-6, StudIS Publishing House, Iaşi, 2013.

[12] Béla Finta, Zsuzsánna Finta, Geometrical Inequalities in Acute Triangle Involving the Medians IV, International Conference "Mathematical Education in the Current European Context", 4th edition, November 22, 2013, Braşov, Romania, pp. 169-174, ISSN 2360-5324, ISSN-L 23605324, StudIS Publishing House, Iaşi, 2014.

[13] Béla Finta, Geometrical Inequalities in Acute Triangle Involving the Medians V, Scientific Bulletin of the "Petru Maior" University of Tirgu Mures, Vol. 11(XXVIII) no. 1, pp. 50-52, ISSNL 1841-9267 (Print), ISSN 2285-438X (Online), ISSN 2286-3184 (CD-ROM), 2014.

[14] Béla Finta, Geometrical Inequalities in Acute Triangle Involving the Medians VI, Scientific Bulletin of the "Petru Maior" University of Tirgu Mures, Vol. 12(XXIX) no. 1, pp. 60-62, ISSNL 1841-9267 (Print), ISSN 2285-438X (Online), ISSN 2286-3184 (CD-ROM), 2015.

[15] Béla Finta, Geometrical Inequalities in Acute Triangle Involving the Medians VII, Scientific Bulletin of the "Petru Maior" University of Tirgu Mures, Vol. 12(XXIX) no. 2, pp. 56-59, ISSNL 1841-9267 (Print), ISSN 2285-438X (Online), ISSN 2286-3184 (CD-ROM), 2015.

[16] Béla Finta, Geometrical Inequalities in Acute Triangle Involving the Medians VIII, Scientific Bulletin of the "Petru Maior" University of Tirgu Mures, Vol. 13(XXX) no. 2, pp. 39-42, ISSNL 1841-9267 (Print), ISSN 2285-438X (Online), ISSN 2286-3184 (CD-ROM), 2016. 